\title{
Déclaration de Corinne Cicéron Bühler
}

\author{
Directrice de la Direction du Droit International Public et Conseillère \\ Juridique du Département Fédéral des Affaires Étrangères Suisse
}

(French original)

C'est un honneur pour moi de participer aujourd'hui à la séance solennelle, en tant que représentante de l'Etat hôte des réunions de la Commission du droit international. J'ai le plaisir de vous adresser, au nom du Conseil fédéral, quelques mots pour marquer le soixante-dixième anniversaire de la Commission du droit international, qui est placé sous le thème ambitieux « Dresser le bilan pour l'avenir ».

Depuis la fin de la guerre froide, le monde est en constante mutation. Les rapports de force au niveau international se modifient dans un contexte marqué par la globalisation et la fragmentation. Les relations internationales ont gagné en importance mais sont aussi devenues plus complexes, notamment en lien avec le climat de volatilité qui prévaut. Le droit international aurait-il fait son temps? Non, bien au contraire. C'est pourquoi il est capital de reconnaître et de souligner son rôle fondamental dans les relations entre États.

Le développement progressif et la codification du droit international sont ainsi essentiels au maintien d'un ordre international stable, juste et pacifique, particulièrement dans un monde confronté à des bouleversements. Le droit international constitue ainsi le garant de relations internationales basées non pas sur le droit de la force mais au contraire sur la force du droit.

En tant que petit Etat, fortement interconnecté, la Suisse a un intérêt marqué au maintien et au renforcement du droit international. Un tel renforcement constitue non seulement un élément fondamental de la Charte des $\mathrm{Na}$ tions Unies mais est également essentiel pour la politique extérieure suisse. Ce constat valait il y a 70 ans déjà. Force est de constater qu'il conserve toute sa pertinence de nos jours.

Aucun pays, aucun acteur sur la scène mondiale n'est en mesure de trouver, seul, les réponses aux défis d'aujourd'hui. Il en va de même pour les questions juridiques, d'où l'importance des travaux de la Commission du droit international.

Si la Commission continue de s'engager pour les sujets de droit international général, tels que le droit des traités ou l'immunité des représentants des Etats, elle s'est déjà saisie de problèmes plus contemporains telles la protection de l'atmosphère, la protection de l'environnement en lien avec les conflits armés 
et la protection des personnes en cas de catastrophe. En effet, la Commission du droit international est appelée à se concentrer également sur le développement progressif de nouvelles règles visant à appréhender les enjeux du monde actuel. La pertinence et l'efficacité du droit international n'en seront ainsi que renforcés. Il est vrai que la Commission a été créée dans le but de promouvoir le développement progressif du droit international et non pas seulement sa codification.

La valeur des travaux de la Commission du droit international n'est plus à démontrer, les projets d'articles de la Commission du droit international jouissant d'une grande autorité dans la pratique et étant souvent interprétés comme des énoncés de droit par les tribunaux nationaux.

C'est donc un grand honneur pour la Suisse d'accueillir à Genève la Commission pour ses travaux et de pouvoir, par ce biais, contribuer à son importante activité. Je souhaite rappeler les propos tenus par mon collègue l'Ambassadeur Jürg Lauber le 21 mai à New York lors de la réunion solennelle : le choix de Genève comme siège des réunions de la Commission permet notamment de garantir la complète indépendance de son activité par rapport à la Sixième Commission, qui siège à New York, et dont le travail est aussi grandement apprécié par la Suisse. La diversité des cultures juridiques propres à ces deux organes constitue un atout pour le développement du droit international. Une présence à Genève assure des synergies avec les nombreuses organisations internationales, les plateformes et les acteurs internationaux qui se trouvent à Genève et exercent une influence sur le quotidien de chacun.

Par ailleurs, la Suisse estime très important que le droit international soit promu non seulement à New York mais également au siège européen des Nations Unies, à Genève. Cela me donne aussi l'occasion de rappeler le Séminaire du droit international qui se tient chaque année à Genève et qui permet à ses participants - fonctionnaires, professeurs et étudiants - de suivre les travaux de la Commission du droit international de très près. Une de mes tâches, en tant que Conseillère juridique du Département fédéral des affaires étrangères, est précisément de suivre le développement et la codification du droit international et je suis particulièrement heureuse que la Commission mène une importante partie de ses travaux en Suisse.

Au nom du gouvernement suisse, je souhaite vous assurer que la Suisse, Etat hôte de la Commission, va continuer à soutenir le travail de cette dernière et à faire le nécessaire pour que ses membres puissent travailler dans le cadre le plus propice au bon déroulement de ses travaux.

Comme indiqué, c'est un grand honneur pour moi d'être parmi vous aujourd'hui pour célébrer cet important anniversaire Je ne doute pas que les 
discussions que vous mènerez aujourd'hui sur le thème « Dresser le bilan pour l'avenir » seront fructueuses et me réjouis déjà de prendre connaissance des résultats de vos réflexions. Il me reste à vous souhaiter d'excellents travaux et vous remercier de votre attention. 